\title{
System Framework for the Design of an Avionics Architecture with Upgrade Potential
}

\begin{abstract}
N. Rao, R. Kusumo, A. K. Sinha, M. L. Scott
The technological growth of 'avionics systems' has outpaced the service-life of aircraft, resulting in avionics upgrade as a preferred cost-effective option to new design. Mid-life upgrade of "avionics systems" by state-of-the-art mission systems has been a challenging engineering task. The complexity of avionics upgrade process is due to the design rigidity of avionics systems architecture. An avionics architecture with growth potential is required to optimise avionics upgrade with state-of-the-art systems. A research program that partially addresses avionics systems upgrade by developing a methodology to design an avionics architecture with in-built growth potential is discussed in this research paper. A system approach' is adopted to develop a methodology that identifies the design parameters that will facilitate design of an avionics architecture with upgrade potential.
\end{abstract}

Keywords: avionics systems, avionics upgrade, system approach, avionics architecture.

\section{Introduction}

During the service life of military aircraft, advances in avionics technology render certain systems onboard either obsolete or of limited capability, compared to a state-of-the-art system. Mid-life upgrade of military aircraft that includes insertion of advanced avionics systems in the "avionics architecture" is a cost-effective option to new design [1]. The major challenge in an avionics upgrade design process is the integration of an advanced system with the systems already onboard. The integration process is governed by the avionics architecture of the aircraft [2].

The architecture for military aircraft is based on a functional format. Flight control, navigation, identification of friend or foe, and communication are the common functional format [3]. The design rigidity of such an architecture format limits the degree to which integration can be achieved. The development of 'multi-functional avionics systems', coupled with architecture rigidity, has made the avionics upgrade process an engineering challenge. A new design approach, named Integrated Modular Avionics (IMA), is an attempt to deal with the current design drawbacks of avionics architecture and to address the problem of technology insertion [4], [5]. The principles on which these concepts are formulated are still premature, and there is no major literature on the subject in the public domain.

A design methodology needs to be developed for an avionics architecture with upgrade potential, from a system perspective - one that will holistically address all design parameters and constraints, including technological insertion [6]. The architecture needs to be in an 'open format', to provide in-built growth potential, and to facilitate insertion of state-of-the-art systems into the architecture on a continuous basis during the service life of the aircraft.

This paper presents a framework for the development of a system methodology to design avionics architecture with upgrade potential.

\section{System Methodology}

A system methodology to study the operational needs and operational environment for deriving the mission requirements of military aircraft was developed by Sinha et al [7]. Based on the derived mission requirements, a Mid Life Upgrade System (MLUS) was structured by Sinha et al [6] to identify the system elements (components, attributes and relationships) and develop the system hierarchy [8]. The MLUS hierarchy has aided the identification of state-of-the art mission systems for mid-life upgrade of in-service military aircraft [9]. The mission systems identified include advanced avionics systems as replacements for obsolete systems on board, or as additional systems to enhance mission capability. The insertion of these state-of-the-art avionics systems on board as part of the upgrade process depends on the "Avionics System Architecture" (ASA) - the platform on which all avionics systems rest.

The design structure of the ASA is based on state-of-the-art technology during the design phase of ASA. As the ASA remains an integral part of the aircraft during its service life, the technological parameters on which the design was based remain static. On the other hand, advances in avionics systems technology continue, resulting in new or modified design parameters. Hence, to facilitate the insertion of advanced systems there is a need for an ASA with Upgrade Potential (ASA-UP) - one that focuses on the design parameters of future avionics systems.

The IMA concept and the methodology for mid-life up-grade analysis of military aircraft provides the foundation to formulate a research program on ASA-UP design methodology. The system methodology developed by Sinha et al [6], [7], [9] can be explored to identify advanced avionics systems. The methodology could then be further explored to identify the ASA-UP design parameters.

In order to develop a methodology that holistically [10] addresses an ASA-UP design, a system structure [6] for avion- 
ics upgrade first needs to be initially formulated. The system structure should facilitate the identification of system elements based on the slated functions of the Avionics Upgrade and System (AUS). Keeping the provisions of technological insertion as the focus, the functions of the AUS to be structured are as follows:

- Identify state-of-the art avionics systems;

- Formulate technological growth parameters;

- Identify the avionics architecture parameters of the aircraft system; and

- Integrate growth and architecture parameters to identify the design parameters of ASA-UP.

The structure of the AUS formulated considering the above functions is presented in Fig. 1 .

\section{System framework}

After conceptualising the avionics upgrade process from a system perspective the framework for the design of an
ASA-UP can be developed. The AUS structure identifies the need for four components - two analysers, an integrator, and a tester and validator - to aid the design of an ASA-UP. The components and their stated functions are as follows:

- Analysers: To provide an analysis of the current architecture and advanced systems, and the identify the architecture and technological growth parameters;

- Integrator: To integrate the architecture and technological growth parameters, and to update the design parameters for an ASA-UP; and

- Tester \& Validator: To test and validate the ASA-UP design parameters for functionality, compatibility and performance.

When the above modules (components) and their functions have been identified, the system framework for the design of an avionics architecture with upgrade potential can be developed. The system framework with the various modules and the functional flow is presented in Fig. 2.

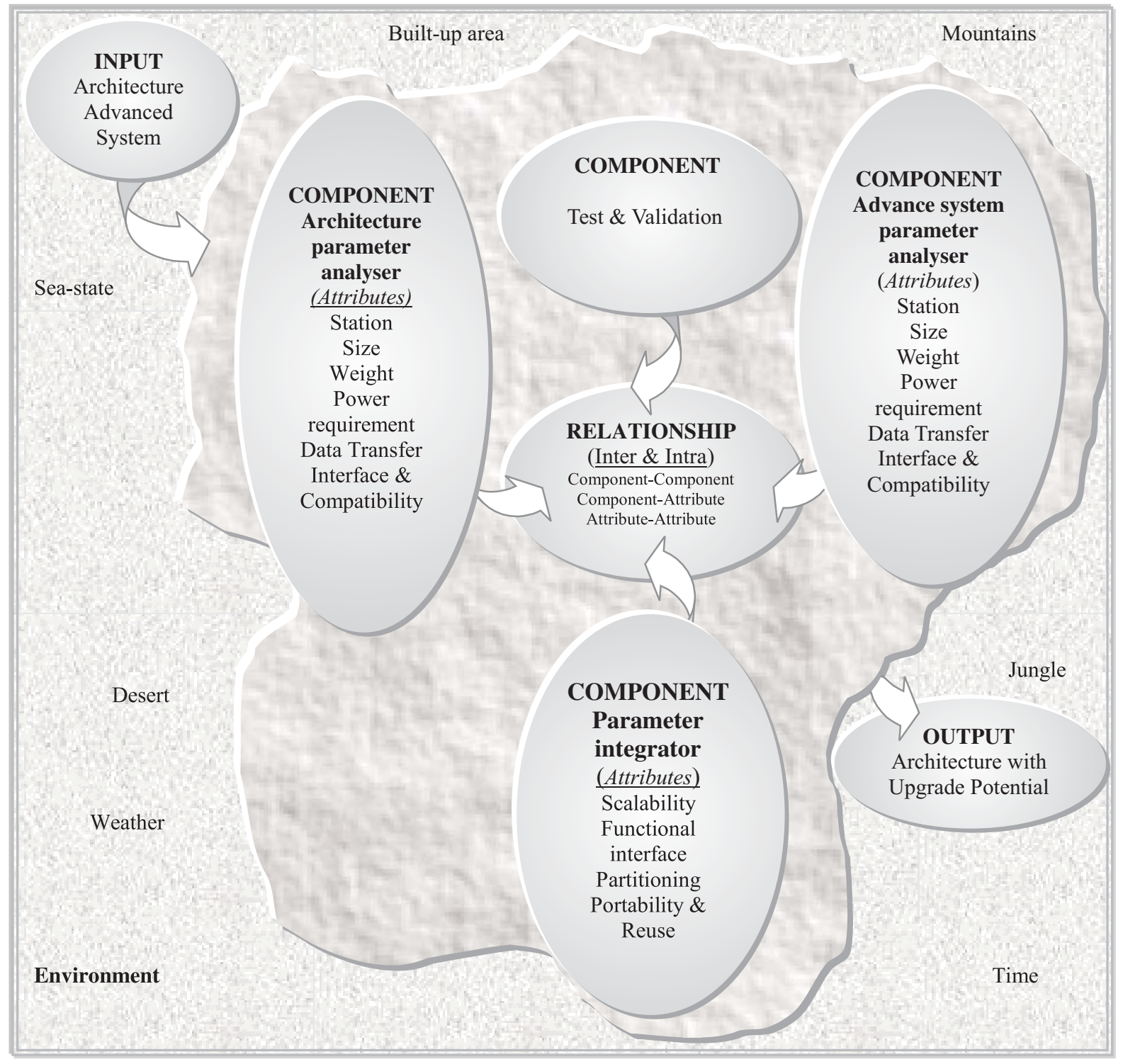

Fig. 1: System structure of an avionics upgrade system 


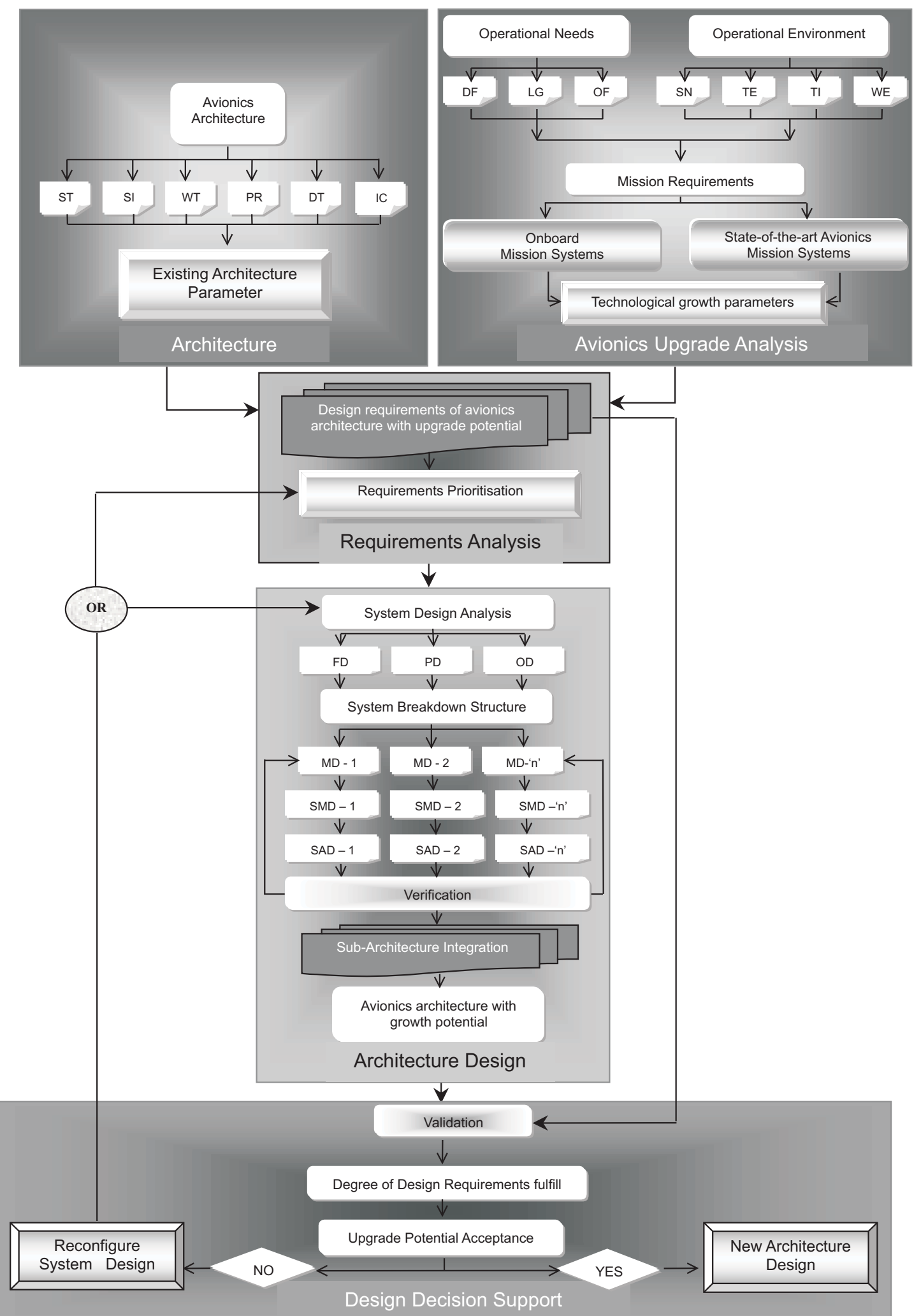

\begin{tabular}{clllll|} 
Legends: & & & & \\
\hline \hline CS: & Costs & DF: & Defensive mission & DT: & Data transfer \\
IC: & Interference \& compatibility & LG: & Logistic mission & MP: & Module parameters \\
OF: & Offensive mission & PR: & Power requirement & SAD: & Sub-architecture design \\
SC: & System capability & SF: & System functionality & SI: & Size \\
SMP: & Sub-module parameters & SN: & Situation & SP: & System performance \\
ST: & Station & TE: & Terrain & TI: & Time \\
WE: & Weather & WT: & Weight & & \\
\hline
\end{tabular}

Fig. 2: System framework for the design of an avionic architecture with upgrade potential 


\section{Results and discussion}

The application of generic system methodology for the identification of advanced mission systems resulted in the following:

- Development of a "System Structure of an Avionics Upgrade System"; and

- Development of a "System Framework for the Design of an Avionics Architecture with Upgrade Potential”.

\section{System structure}

The system structure that has been developed provides a system perspective of an Avionics Upgrade problem. It identifies the components of the problem and the functional requirements that need to be addressed for a holistic solution. An interrelationship analysis of the components and their attributes (functional requirements) provides the methodology to integrate the design parameters of the architecture and advanced avionics systems.

\section{System framework}

The system framework identifies four analysis modules Architecture analysis, Avionics upgrade analysis, Architecture design and Decision support. Each of these modules comprises various sub-modules that aid analysis and decision support. The sub-module frameworks need to be further developed in detail for functionality of the modules. The system framework caters for feedback loops to optimise the design.

\section{Conclusion}

The system approach adopted for developing the framework for the design of an Avionics Architecture with Upgrade Potential provides an avenue for a holistic analysis of the problem. The methodology developed addresses all design parameters that need to be considered in the design process.

\section{Reference}

[1] Little, R.: Advanced Avionics for Military Needs. Proceedings of Royal Aeronautical Society on Avionics in the Future Land-Air Battle, London, U.K., Hamilton Place, 12 December 1990, pp. 11.1-11.10

[2] Morgan, D. R.: Military Avionics Twenty Years in the Future. AIAA/IEEE Digital Avionics Systems Conference,
5-9 November, Massachusetts, Cambridge, 1995, pp. $483-490$

[3] Rushby, J.: Partitioning in Avionics Architectures: Requirements, Mechanisms, and Assurance. NASA/CR-1999-209347, California 1999

[4] Giddings, B. J.: Some Fundamentals of Integrated Modular Avionics. Proceedings of Royal Aeronautical Society, April, Maryland, Annapolis, 1999, pp. 4.1-4.7

[5] Design Guidance for Integrated Modular Avionics. ARINC Specification 651, No. 1991, Aeronautical Radio Inc., Annapolis, MD

[6] Sinha, Bil, Scott: Design pf Payloads for Mid-life Upgrade of Maritime Helicopters: Stages I, II, III and IV. Third Australian Pacific Vertiflite Conference on Helicopter Technology, 12-14 July, ACT, Canberra 2000

[7] Sinha, Kem, Wood: A system approach to Helicopter Modifications for Multi-mission Roles. First Australian System Conference, WA, Perth 2000

[8] Blanchard, B. S., Fabrycky, W. J.: Systems Engineering and Analysis. Prentice Hall, New Jersey, 1990

[9] Sinha, Bil, Scott: Design of Payloads for Mid-life Upgrade of Maritime Helicopters: Stages V and VI. Presented at the International Conference on Systems Thinking in Management, 08-10 November, Melbourne, VIC, 2000

[10] Flood, R. L., Jackson, M. C.: Creative Problem Solving Total Systems Intervention. John Wiley and Sons, New York 1991

Nagendra Rao

e-mail: nagendra.rao@rmit.edu.au

Raden Kusumo

Department of Aerospace Engineering 226 Lorimer Street Fishermens Bend Port Melbourne, VIC 3207, Australia

Dr. Arvind Kumar Sinha Murray L. Scott

The Sir Lawrence Wackett Centre for Aerospace Design Technology Department of Aerospace Engineering Royal Melbourne Institute of Technology GPO Box 2476V, Melbourne VIC 3001, Australia 